\title{
MetamorHockey: A Projection-based Virtual Air Hockey Platform Featuring Transformable Mallet Shapes
}

\author{
Shun Ueda \\ shun.ueda.t5@dc.tohoku.ac.jp \\ Tohoku University \\ Sendai, Japan
}

\author{
Shingo Kagami \\ swk@ic.is.tohoku.ac.jp \\ Tohoku University \\ Sendai, Japan
}

\author{
Koichi Hashimoto \\ Tohoku University \\ Sendai, Japan
}

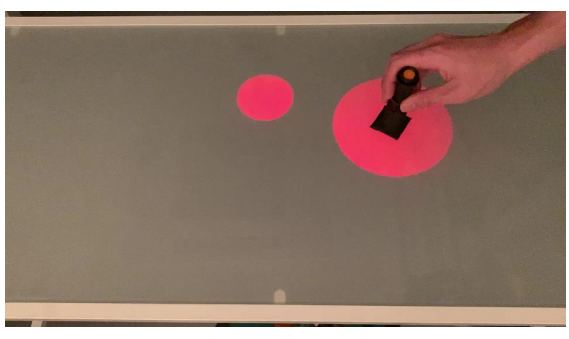

(a) Resized mallet

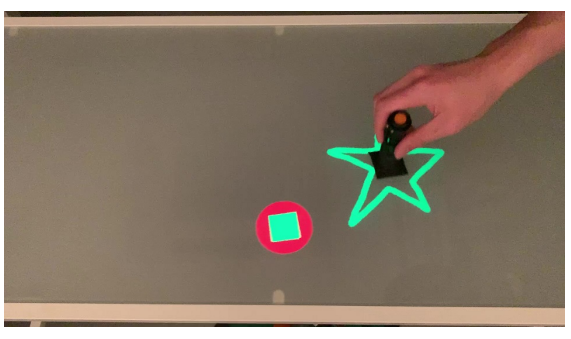

(b) Mallet shape defined by drawing

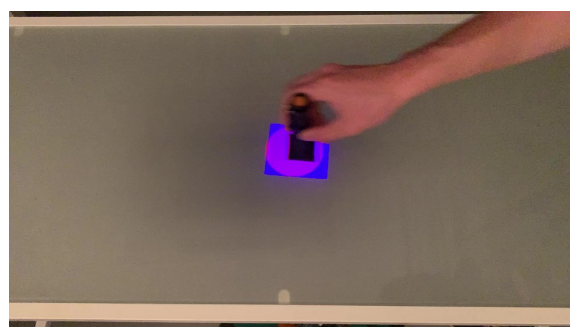

(c) Disabled collision between mallet and puck

Figure 1: Snapshots of the proposed system displaying different shapes of air hockey mallets.

\begin{abstract}
We propose a novel projection-based virtual air hockey system in which not only the puck but also the mallet is displayed as an image. Being a projected image, the mallet can freely "metamorphose" into different shapes, which expands the game design beyond the original air hockey. We discuss possible scenarios with a resizable mallet, with mallet shapes defined by drawing, and with a mallet whose collision conditions can be modified. A key challenge in implementation is to minimize the latency because the direct manipulation nature of the mallet positioning imposes a higher demand on latency than the puck positioning. By using a high-speed camera and a high-speed projector running at $420 \mathrm{fps}$, a satisfactorily quick tracking became possible such that we feel a projected mallet head to be an integral part of a mallet held by hand.
\end{abstract}

\section{CCS CONCEPTS}

- Human-centered computing $\rightarrow$ Interaction devices.; • Computing methodologies $\rightarrow$ Mixed / augmented reality.

\section{KEYWORDS}

high-speed projection, interaction

\section{ACM Reference Format:}

Shun Ueda, Shingo Kagami, and Koichi Hashimoto. 2021. MetamorHockey: A Projection-based Virtual Air Hockey Platform Featuring Transformable Mallet Shapes. In Special Interest Group on Computer Graphics and Interactive Techniques Conference Emerging Technologies (SIGGRAPH '21 Emerging

This work is licensed under a Creative Commons Attribution International 4.0 License.

SIGGRAPH '21 Emerging Technologies, August 9-13, 2021, Virtual Event, USA (c) 2021 Copyright held by the owner/author(s).

ACM ISBN 978-1-4503-8364-6/21/08.

https://doi.org/10.1145/3450550.3465341
Technologies ), August 9-13, 2021, Virtual Event, USA. ACM, New York, NY, USA, 4 pages. https://doi.org/10.1145/3450550.3465341

\section{INTRODUCTION}

Air hockey is a sport that is played between two competing players who try to put a puck in the opposing player's goals. A player hits the puck with a mallet (also called a striker or a paddle) on a table of which the surface friction is designed to be low, usually by blowing air from the tabletop. Bisides being a popular professional sport, it is also popular as a casual recreation game played in a gaming arcade, or even at home with a down-sized setup, because of its simple but exciting nature.

For such amusement purposes, air hockey is sometimes combined with special lighting and sound effects to enhance the excitement and increase the engagement in the game. The efforts made along this direction include the use of virtual and augmented reality technologies. An early attempt [Ohshima et al. 1998] employed optical see-through head-mounted displays (HMDs) to realize air hockey with a virtual puck. More recent efforts utilized video seethrough HMDs to modify the appearance of a physical puck [Chinen et al. 2017] or a projection-mapping display to enable a play with a mixture of physical and virtual pucks [Sony Corporation 2018]. Other example use cases of projection-based augmented reality techniques include instant replay of a fast physical movement of a puck in a reduced speed [Nii et al. 2006] and presentation of a predicted path of a physical puck [Arai et al. 2011].

Although the use of virtual and augmented pucks in air hockey has been explored in the above-mentioned efforts, the mallets have always been physical ones. If we can replace a physical mallet with a virtual one, further expansions of the game design space is expected. A motivating example is Grid Air Hockey [Ono 2018], in which players are able to design their own air hockey field with physical blocks and choose a physical mallet among those with 
different shapes, although the choice is limited to a predetermined set.

Displaying a virtual mallet image in a fast air hockey play requires a significantly lower latency than displaying a virtual puck does, which explains why implementation of such a system has been avoided. Here, we refer by latency to the time interval between the instant when a physical action occurs and the instant when the displayed image responds to it. The reason of this higher demand on latency is twofold. Firstly, a spontaneous movement of a player's hand is difficult to predict, whereas a puck is ruled by a much simpler dynamics and latency hiding through prediction is relatively easy.

Secondly, because the ideal position of a displayed virtual mallet is clearly indicated by the hand position of a player, the player tends to perceive the position error sensitively. This can be understood by recalling that displaying a virtual mallet precisely at a finger or a stylus position is essentially the same as implementing a dragging interaction. It is known that users are able to discriminate latencies as low as a few milliseconds when they experience dragging interaction in direct touch [Jota et al. 2013; $\mathrm{Ng}$ et al. 2012] or direct stylus-pointing [ $\mathrm{Ng}$ et al. 2014] devices, whereas tapping or scribbling interactions do not impose such a high demand on latency.

In this paper, we implement and demonstrate a projection-based air hockey platform in which both of the puck and the mallet are represented by projected images. To minimize the latency, we use a high-speed camera and a high-speed projector [Kagami and Hashimoto 2018] that enables fast display updates, which contributes to lowering the latency. Running at around $420 \mathrm{fps}$, the system achieves an accurate registration of a mallet image to a stylus-like pointing device held by a player's hand, which contributes to provide a strong sense of possession of the mallet. We also discuss possible expansions of air hockey game design through the use of transformable pucks and mallets.

\section{EQUIPMENTS}

Figure 2 shows an overview of the prototype MetamorHockey system. This prototype includes a small hockey field with a single puck and single mallet for a single user trial.

A rear-projection screen table is used as an air hockey field, onto which a high-speed projector [Kagami and Hashimoto 2018] projects an image. A player holds a stylus-like pointing device that assumes the role of a mallet handle. This pointing device is equipped with an infrared (IR) LED, the light emitted from which is tracked by a FLIR FL3-U3-13Y3M-C USB-3 camera. The whole system is controlled by a laptop PC with an Intel Core i7-10710U processor.

The projector is equipped with a Texas Instruments DLP7000BFLP XGA Digital Micromirror Device (DMD) with a custom controller. The controller implements a homography warping engine implemented in an FPGA that applies up to 2,470 transformations per second to a preloaded binary patterns before sending them to the DMD chipset.

The projector is placed under the rear-projection screen table so that players can view the projected image on the tabletop. The camera with an IR-pass filter is also placed under the table to capture,

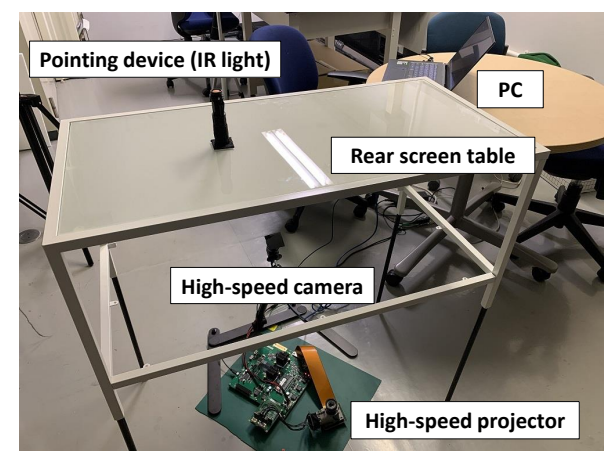

Figure 2: Overview of the prototype system.

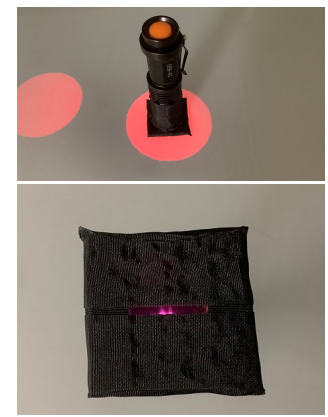

(a) Pointing device

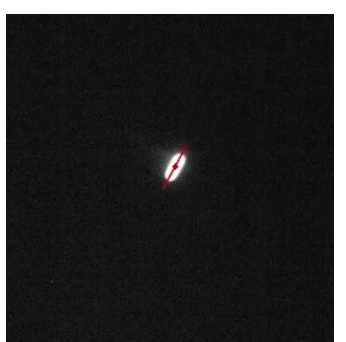

(b) Image captured by the camera
Figure 3: Pointing device design (a) and an image from the high-speed camera (b). In (b), The red line is the principal axis of the ellipsoid spot, and the red point is its center of mass.

through the screen, the light from the pointing device put upon the table.

The pointing device is composed of a Stork Corp SA-013 IR flash light $(850 \mathrm{~nm}, 5 \mathrm{~W})$ and a lens cover made with a 3D printer. The lens cover has a slit at its center and this side is the bottom surface of the mallet handle that contacts with the table surface. The camera observes the IR light through this slit, yielding a bright ellipsoid spot in the image as shown in Figure 3.

\section{VISUALIZATION OF PUCK AND MALLET}

\subsection{Tracking the Pointing Device}

Before the system initialization, we carry out calibration between the projector image and the camera image. During the calibration process, the IR-pass filter on the camera lens is removed and a chessboard image projected onto the screen is observed by the camera. From the corresponding corner points in the projector and camera images, the mapping between these two images is obtained.

The IR light spot is tracked by detecting the center of mass of bright pixels in the camera image. Then the principal axis of the spot is computed to determine the orientation of the pointing device. Because the tracking is limited to the 2D rigid motion, 3D actions such as holding the puck down from the above to stop it cannot be realized. 
Our system runs at as fast as $420 \mathrm{fps}$. In spite of that, we found that rendering a mallet image simply at the point corresponding to the detected IR light spot suffers from a perceivable delay when the device is moved fast. Therefore, we implemented a simple prediction step in which a constant velocity model is assumed. Owing to the high frame rate operation, this simple model works well to hide the latency apparently without introducing perceivable prediction errors.

In Figure 5, we compare the result when running at $420 \mathrm{fps}$ (Figure $5 \mathrm{a}$ ) and the result when the display update rate is intentionally reduced to $60 \mathrm{fps}$ (Figure $5 \mathrm{~b}$ ). Note that the frame rate of image capturing and image processing is kept at $420 \mathrm{fps}$ in both of these results, and thus the difference between them is attributed only to the display update rate. Overall, the position errors between the center of the projected mallet head and the center of the mallet handle in Figure 5a are smaller than those in Figure 5b. We can observe a remarkable error especially at $t=0.05[\mathrm{~s}]$ in Figure $5 \mathrm{~b}$, where the pointing device started to move.

As an informal subjective evaluation by the authors, with the 60 -fps update rate, we feel that the displayed mallet head follows the mallet handle. In contrast, with the 420 -fps update rate, we feel that the mallet head is an integral part of a mallet, despite the fact that the still images in Figure 5a show there remain tracking errors.

\subsection{Physics Simulation}

The positions and orientations of the displayed puck and mallet are computed using a physics simulator Box2D. The simulated 2D space is defined to be a miniature version of the projector image.

Once the predicted position and orientation of the IR light spot are obtained and mapped into the simulated space, we accordingly set the linear and angular velocities of the mallet in the simulator. Then the physics simulation including the puck and the field boundaries is updated. The simulation results are used to render the images of the puck and the mallet in the projector image space.

In addition to mathematically defined shapes such as circles and polygons, any shapes drawn with a painting software can be imported. In the Box2D simulation, a drawn shape is defined by a set of small circular kinematic objects attached to the points that comprise the shape.

\subsection{Implementation Notes}

The projector we use has a distinct architecture to enable lowlatency projection with a relatively low-cost hardware. Instead of receiving a high frame rate video input, it has a capability of applying fast warps to preloaded images or a video rate input to generate projected images quickly adaptable to real-world motion.

Because it was designed specifically focusing on projection mapping onto a moving planar surface [Kagami and Hashimoto 2019], the warping capability implemented within the projector is limited to a single homography transformation at a time.

Displaying two fast moving objects, the mallet and the puck, is implemented in a time division multiplexing manner. Figure 4 shows the sequence diagram of our implementation. While the visual tracking and the physics simulation updating are carried out at $420 \mathrm{fps}$, the puck image appears only at even-numbered frames and the mallet does only at odd-numbered frames. Therefore, in

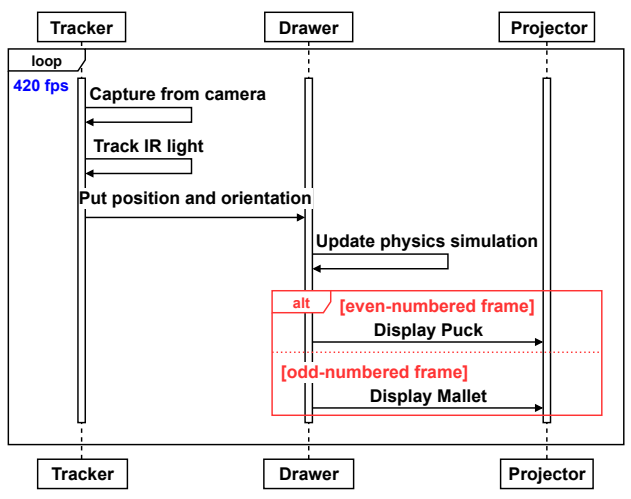

Figure 4: Sequence diagram of the operation.

a strict sense, the frame rate of our display is a half of $420 \mathrm{fps}$. Nevertheless, this does not impact the latency because every display update is governed by the most recent motion detection.

\section{GAME DESIGN EXAMPLES}

The transformability of the mallet appearance including its shape expands the possibility of air hockey beyond its original game design.

An obvious example is to change the shape or the size of a mallet to control the difficulty of game. In general, a game play become easier with a larger mallet. Therefore, it is sensible to resize the mallet in accordance with the proficiency level of the player. It could be also possible to change the size during a game in accordance with gained or lost points.

The capability of defining an arbitrary mallet shape by drawing allows infinite possibilities in game design. For example, the design in Figure 6a allows catching and caging of a puck as well as hitting. The design in Figure $6 \mathrm{~b}$ allows accelerated hitting by rotating a bat-like shape around the handle.

While some designs offer advantages, other designs may introduce difficulties in playing. Figure $6 \mathrm{c}$ is a design composed of two disconnected parts that revolve around the handle at a predefined speed. With this mallet, a player must make a timely hit at a right moment considering both of puck and mallet motions. The opposing player may try to strike a shot through the gap between the two parts. A game design treating a set of different mallet shapes like a deal or a hand in a card game may be possible, in which a player must schedule the use of the mallet set strategically.

Collision detection between a puck and a mallet may be redefined such that it is conditioned on the states of them. Imagine that a player can freely choose a mallet shape during a game while a puck randomly changes its shape, and collision occurs only when the puck and the mallet has the same shape; otherwise the puck passes through the mallet as in Figure 1c. Another possibility in a similar direction is to modify physical properties of mallets such as a restitution coefficient and friction constants.

\section{CONCLUSION}

We have presented a projection-based virtual air hockey system in which the mallet as well as the puck is replaced by a projected image 

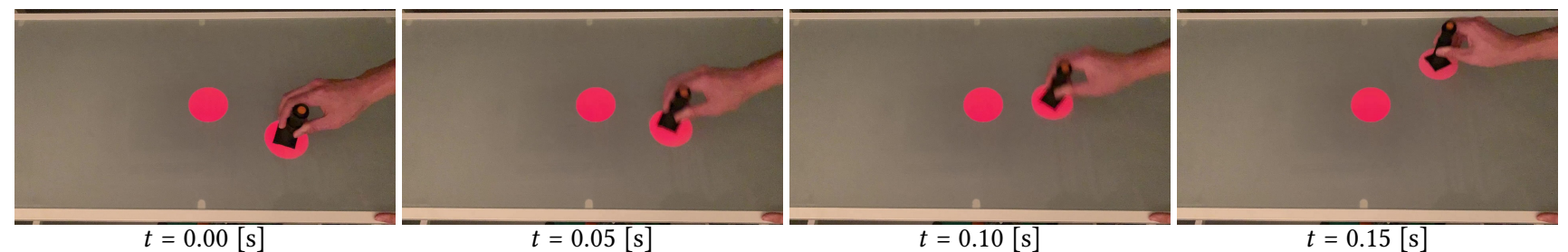

(a) The projection rate is around $420 \mathrm{fps}$.

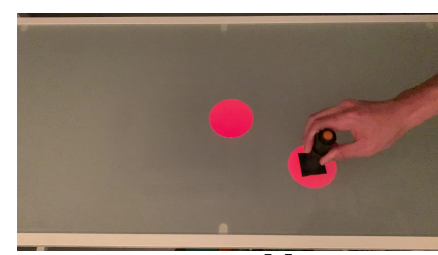

$t=0.00[\mathrm{~s}]$

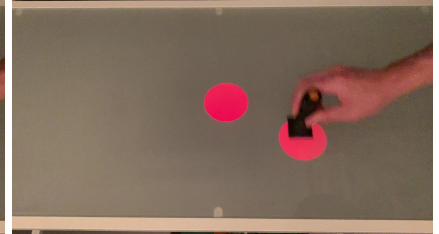

$t=0.05[\mathrm{~s}]$

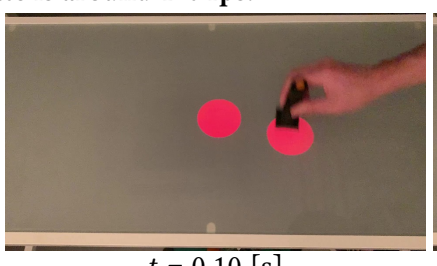

$t=0.10[\mathrm{~s}]$

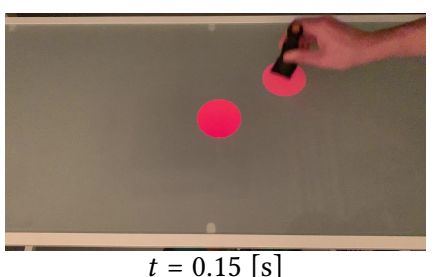

$t=0.15[\mathrm{~s}]$

(b) The projection rate is around $60 \mathrm{fps}$.

Figure 5: Snapshot sequences from MetamorHockey. Projection control at 420fps (top row) offers faster motion adaptation than at 60 fps (bottom row).

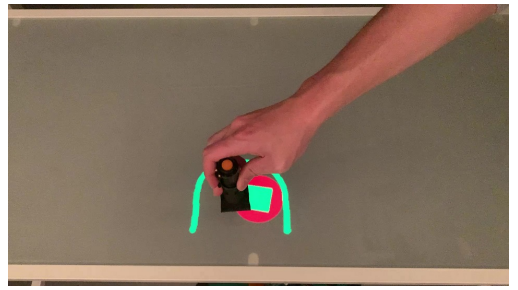

(a) Basket-like shape

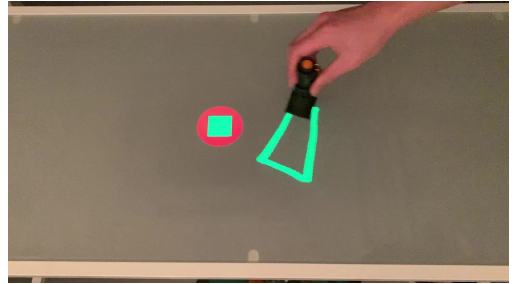

(b) Bat-like shape

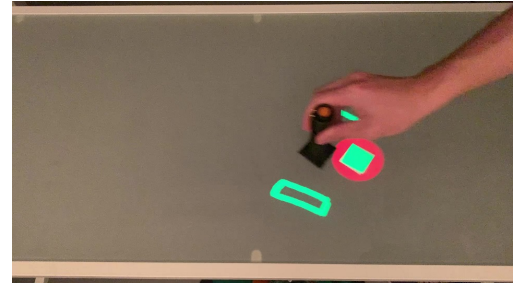

(c) Shape with disconnected components

Figure 6: Mallet design examples

and thus its appearance is transformable. With an implementation using a high-speed camera and a high-speed projector, a strong sense of possession of a mallet is offered although it does not have a physical entity. We discussed how the air hockey game design can be expanded through the transformability of the mallet.

Our current prototype comes with a downsized hockey table and only a single player can experience an interaction with a puck and a mallet. Building a scaled-up version as future work could be an option, but we also plan to extend our approach to a networkconnected air hockey system over a distance [Mueller et al. 2006], which will contribute to development of social entertainment interactions conforming to the new normal.

\section{ACKNOWLEDGMENTS}

Part of this work was supported by JSPS Grand-in-aid 19H04146, 16H06536, and JST ACCEL JPMJAC1601.

\section{REFERENCES}

Yusuke Arai, Shingo Kagami, and Koichi Hashimoto. 2011. Experimental studies on prediction in air hockey games. In 2011 IEEE/SICE International Symposium on System Integration (SII). 112-117.

Yuta Chinen, Tsuneya Kurihara, and Takuya Nojima. 2017. A study on Augmented Air Hockey using video-see-through HMD. In Entertainment Computing Symposium 2017 (EC2017). 266-274. (in Japanese).
Ricardo Jota, Albert Ng, Paul Dietz, and Daniel Wigdor. 2013. How Fast is Fast Enough? A Study of the Effects of Latency in Direct-Touch Pointing Tasks. In SIGCHI Conference on Human Factors in Computing Systems (CHI '13). 2291-2300.

Shingo Kagami and Koichi Hashimoto. 2018. A full-color single-chip-DLP projector with an embedded 2400-fps homography warping engine. In ACM SIGGRAPH 2018 Emerging Technologies (SIGGRAPH '18). Article no.1.

Shingo Kagami and Koichi Hashimoto. 2019. Animated Stickies: Fast Video Projection Mapping onto a Markerless Plane through a Direct Closed-Loop Alignment. IEEE Trans. Vis. Comput. Graph 25, 11 (2019), 3094-3104.

Florian 'Floyd' Mueller, Luke Cole, Shannon O'Brien, and Wouter Walmink. 2006. Airhockey over a Distance: A Networked Physical Game to Support Social Interactions. In 2006 ACM SIGCHI International Conference on Advances in Computer Entertainment Technology (ACE '06). 70-es.

Albert Ng, Michelle Annett, Paul Dietz, Anoop Gupta, and Walter F. Bischof. 2014. In the Blink of an Eye: Investigating Latency Perception during Stylus Interaction. In SIGCHI Conference on Human Factors in Computing Systems (CHI '14). 1103-1112.

Albert Ng, Julian Lepinski, Daniel Wigdor, Steven Sanders, and Paul Dietz. 2012. Designing for Low-Latency Direct-Touch Input. In 25th Annual ACM Symposium on User Interface Software and Technology (UIST '12). 453-464.

Hideaki Nii, Jay Summet, Yong Zhao, Jonathan Westhues, Paul Dietz, Shree Nayar, John Barnwell, Michael Noland, Vlad Branzoi, Erich Bruns, Masahiko Inami, and Ramesh Raskar. 2006. Instant Replay Using High Speed Motion Capture and Projected Overlay. In ACM SIGGRAPH 2006 Emerging Technologies (SIGGRAPH '06). 111-es.

Toshikazu Ohshima, Kiyohide Satoh, Hiroyuki Ymamoto, and Hideyuki Tamura. 1998. AR2Hockey : A case study of collaborative augmented reality. IEEE Virtual Reality Annual International Symposium (1998), 268-295.

Yoshinaka Ono. 2018. GRID AIR HOCKEY. Retrieved February 23, 2021 from https://yoshinakaono.com/GRID-AIR-HOCKEY

Sony Corporation 2018. A(i)R Hockey. Retrieved February 23, 2021 from https: //www.sony.net/SonyInfo/design/stories/AiRhockey/ 